\title{
Effects of superheated steam processing on common buckwheat grains: Lipase inactivation and its association with lipidomics profile during storage
}

\section{Lijuan Wang}

China Agricultural University

\section{Libo Wang}

China Agricultural University

Ju Qiu

Chinese Academy of Agricultural Sciences Institute of Food and Nutrition Development

Zaigui Li ( $\square$ lizg@cau.edu.cn)

China Agricultural University https://orcid.org/0000-0002-4921-6414

\section{Research}

Keywords: Lipidomics profile, Lipase, Superheated steam, Buckwheat, Hydrolytic rancidity

Posted Date: May 5th, 2020

DOI: https://doi.org/10.21203/rs.3.rs-26175/v1

License: (c) (i) This work is licensed under a Creative Commons Attribution 4.0 International License. Read Full License

Version of Record: A version of this preprint was published at Journal of Cereal Science on September 1st, 2020. See the published version at https://doi.org/10.1016/j.jcs.2020.103057. 


\section{Abstract}

Background: Buckwheat deteriorates easily during storage, resulting loss of nutrients, rancidity flavor and poor consumer acceptability. Superheated steam (SS) was used to inactivate lipase of common buckwheat grains in this study, in order to retard lipid hydrolytic rancidity and maintain lipid nutrition of common buckwheat.

Methods: Buckwheat grains were treated with SS at $110-200^{\circ} \mathrm{C}$, for $0-7$ min and SS treatment parameters were optimized by moisture content and lipase activity. The changes in free fatty acid (FFA) and lipase activity of SS-treated and untreated buckwheat during 12 weeks storage at $4{ }^{\circ} \mathrm{C}, 25^{\circ} \mathrm{C}$ and $50^{\circ} \mathrm{C}$ were determined. Meanwhile, the effects of SS treatment on fatty acid compositions and lipidomics profile of buckwheat before and after storage were also evaluated. Moreover, the associations of hydrolytic rancidity with lipase activity and lipidomics profile were analyzed.

Results: SS processing at $170{ }^{\circ} \mathrm{C}$ for 5 min was proved to be an effective method for buckwheat stabilization. Better stabilities based on lower FFA accumulation and lipase activity were observed in SStreated buckwheat samples during storage. Meanwhile, SS could suppress oxidation of unsaturated fatty acids (UFA) in buckwheat, significantly retard the increase of saturated fatty acids (SFA) and the decrease of polyunsaturated fatty acids (PUFA) during storage. Moreover, the lipidomics profile results indicated that SS processing could retard the increased hydrolysis and oxidation of sulfoquinovosyl diacylglycerol (SQDG), phosphatidylethanolamine (PE), phosphatidylserine (PS) and lysophosphatidic acid (LPA) during storage.

Conclusion: Thus, SS processing could effectively inactivate lipase, suppress UFA oxidation, change glycerolipids (GLs) and glycerophospholipids (GPs) subclass metabolism, and consequently retard hydrolytic rancidity and lipid nutrition loss of buckwheat during storage. This work was first time to demonstrate the application of SS processing for the effective quality control of buckwheat during storage.

\section{Background}

Consumers have been increasingly interested in buckwheat due to its unique nutritional compositions and various health benefits [1]. Although the contents of lipids are less than those of starch and protein, they play a key role in nutritional and functional significance [2]. Lipids are generally categorized into eight classes: fatty acids, glycerolipids (GLs), glycerophospholipids (GPs), sphingolipids, sterol lipids, prenol lipids, saccharolipids, and polyketides [3, 4]. GLs and GPs as the structural elements of cell membranes play the unique role in stabilizing cell membranes and protecting membrane lipids from hydrolytic enzymes [5]. Dietary GPs have beneficial effects on a range of human diseases including coronary heart disease, cancer and inflammation [6]. Evidence suggests that a high intake of saturated fatty acids (SFA) from the diet may be associated with elevated cardiovascular disease risk, the change in SFA/unsaturated fatty acid (UFA) ratio may contribute to improve human health [7]. Common 
buckwheat (Fagopyrum esculentum) as one of the main cultivated buckwheat species in the world contains $8-11 \%$ phospholipids (of total lipids in buckwheat), lower SFA amounts and higher essential fatty acid (mainly linoleic acid), which may promote its positive health effects [8].

Although the lipid content in buckwheat ranges from 1.9-3.2\%, it mainly contributes to easy deterioration of buckwheat during storage [8]. The content of free fatty acid (FFA) can be increased significantly and exceeded limit within short storage duration, resulting in rancidity flavor and poor consumer acceptability of buckwheat flour. UFA are easily oxidized to produce hydroperoxides and other harmful substances. It has been reported that lipid degradation and oxidation plays an important role in buckwheat quality deterioration during storage [9-11]. However, the detailed lipid degradation pathways mechanisms are unclear. Meanwhile, the lipidomics profile of buckwheat is unavailable, which limits the further study and applications of buckwheat.

The lipase activity is an important factor in grain quality deterioration, because it not only affects generation of FFA from lipids hydrolytic rancidity by enzymatic reaction of lipases [12], but also affects subsequent fatty acid oxidation and degradation, involved with the formation of conjugated hydroperoxy fatty acids by lipoxygenase or autoxidation, and then further broken down (non-enzymatic reaction) or oxygenation by peroxidase [13]. Suzuki et al. [9] suggested that lipase activity is relevant to lipid degradation in quality deterioration of buckwheat flour. Moreover, lipase is more thermally stable than lipoxygenase, and lipoxygenase exhibits little activity at the low moisture content of stored grain [14]. Therefore, inactivation of lipase is the common goal for most heat treatment to lengthen the shelf life of cereal grains [14]. However, to our best knowledge, the lipase inactivation methods of buckwheat and their effects on the buckwheat quality is still rarely studied.

To prolong the shelf life and maintain the high quality of cereal grains, various heat treatments designed to inactivate enzymes have been the primary means for cereals processing and storage, such as superheated steam (SS), hot air, infrared heating and microwave [15-19]. SS treatment has became a novel thermal stabilization technology suitable for food processing, because of its several advantages. Firstly, the higher temperature together with higher enthalpy of SS than saturated steam and hot air processing at the same pressure leads to more efficient heating [20]; secondly, high heat penetration of SS can not only increase the temperature of food rapidly, but also result in a reverse moisture transfer induced by the condensation and evaporation of moisture on the food [21, 22]; thirdly, an oxygen-free environment can significantly reduce oxidative degradation reactions during SS processing [22]. Recently, SS has been found to be effective for inactivate enzymes: peroxidase and lipolytic enzymes of wheat bran at $170^{\circ} \mathrm{C}$ within $7 \mathrm{~min}$ [18]; peroxidase of oat groats at $110^{\circ} \mathrm{C}$ for 10 and $14 \mathrm{~min}$ [23]; lipolytic enzymes of brown rice at a temperature above $125^{\circ} \mathrm{C}$ within 2 min [24]. However, there are no detailed studies about the effects of SS processing on buckwheat, especially its effects on lipolytic enzymes inactivation and lipidomics profile.

Given that lipase activity plays an important role in the quality deterioration of buckwheat, we hypothesized that SS treated buckwheat grains prior to storage would inactivate lipase and thus stabilize 
buckwheat during storage. The aim of this study were to (1) investigate the real effects of SS treatment on lipase inactivation in buckwheat by a quantification of lipase activity under different SS-treated conditions; (2) determine the changes in hydrolytic rancidity during different storage conditions of SStreated buckwheat compared with untreated buckwheat; (3) analyze the effect of SS treatment on the lipidomics profile of buckwheat before and after storage.

\section{Methods}

\section{Materials}

Dehulled common buckwheat grains (Chiqiao 1\#, main cultivar of common buckwheat in China) were obtained from the Chifeng Academy of Agriculture and Animal Husbandry Sciences in December, 2018 (Inner Mongolia, China). The initial moisture content of the buckwheat grain was $12.28 \%$. Highperformance liquid chromatography (HPLC)-grade acetonitrile, isopropyl alcohol, and methanol were purchased from Merck (Darmstadt, Germany). Other chemicals used were either analytical or reagent grade.

\section{Optimization of SS treatment}

The moisture content of buckwheat grains was adjusted to $20.0 \%$ (w.b.) by tempering for further SS treatment according to the reported method of Head et al. [25]. Buckwheat grains with a given mass of distilled water in a sealed bag were preserved at $20-25^{\circ} \mathrm{C}$ for $12 \mathrm{~h}$ to guarantee the total absorption of water. Buckwheat grains were treated in an SS processing system developed by the Laboratory of Cereal Science at China Agricultural University, Beijing, China, based on our previous method and schematic diagram [21]. The tempered buckwheat grains (about $300 \mathrm{~g}$ ) were scattered on the metal mesh sample tray in a uniform thin layer $(2-3 \mathrm{~mm})$ and conveyed into the processing chamber. The steam velocity of SS was $15.0 \mathrm{~m}^{3} / \mathrm{h}$, and the temperature was set at $110^{\circ} \mathrm{C}, 140{ }^{\circ} \mathrm{C}, 170^{\circ} \mathrm{C}$ and $200{ }^{\circ} \mathrm{C}$. The processing time ranged from 1 to $7 \mathrm{~min}$ at $2 \mathrm{~min}$ intervals. The buckwheat grains were cooled to room temperature by spreading out after SS treatments, and then were used to analyze moisture and lipase activity immediately. The buckwheat grains without SS treatment were considered as the control. All experiments were conducted in triplicate.

\section{Moisture content}

Buckwheat grains were ground by a high-speed grinding mill (HY-04A, Beijing Huanyatianyuan Instrument Co., Ltd, Beijing, China) and passed through a 60 mesh sieve. The moisture content of ground buckwheat was determined using a standard method (approved method 44-19.01; AACC International, 2017). All measured response variables were reported on a dry weight basis (d.b.).

\section{Determination of lipase activity}

The lipase activity of ground buckwheat was determined according to the previous study of Jha et al. [26] by using glycerol trioleate as a substrate with phosphate buffer of $\mathrm{pH} 7.4$ and expressed as $\mathrm{mg}$ of $0.01 \mathrm{M}$ 
$\mathrm{KOH} / \mathrm{g}$ of buckwheat.

\section{Storage studies}

After SS treatment at $170{ }^{\circ} \mathrm{C}$ for $5 \mathrm{~min}$ as a optimized results, the buckwheat grains were sealed in plastic bags and stored at $4{ }^{\circ} \mathrm{C}, 25^{\circ} \mathrm{C}, 50^{\circ} \mathrm{C}$ in temperature controlled incubators for 12 weeks. After $2,4,8$ and 12 weeks, samples were withdraw from the storages, ground and sieved as described in section of moisture content. The ground buckwheat flour were re-packaged in plastic bags and stored at $-20{ }^{\circ} \mathrm{C}$ until further FFA, lipase activity, fatty acid compositions and lipidomics profile analysis. The untreated buckwheat grains were also stored and analyzed as the control.

\section{Free fatty acids value}

FFA values were determined according to the method of Zhao et al. [27]. The value was expressed as mg $\mathrm{KOH}$ which was consumed by neutralizing FFA in $100 \mathrm{~g}$ ground buckwheat on a dry weight basis (mg $\mathrm{KOH} / 100 \mathrm{~g}$ d.b.). These measurements were carried out in triplicate.

\section{Fatty acid composition}

Buckwheat flour $(0.5 \mathrm{~g})$ were mixed with $1 \mathrm{~mL}$ toluene, $2 \mathrm{~mL}$ sulfuric acid in methanol ( $\mathrm{v} / \mathrm{v}=1: 99)$, then left overnight in a water bath at $50{ }^{\circ} \mathrm{C}$. After cooling to room temperature, the mixture was further mixed with sodium chloride $(5 \mathrm{~mL}, 5 \% \mathrm{w} / \mathrm{v})$ and extracted with hexane twice. The hexane layer was collected and washed with $4 \mathrm{~mL}$ of $2 \%(\mathrm{w} / \mathrm{v}) \mathrm{KHCO}_{3}$-water solution. The upper layer of hexane containing the fatty acid methyl esters was evaporated with $\mathrm{N}_{2}$, and dissolved in $1 \mathrm{~mL}$ hexane for the determination of fatty acids composition using an Agilent 7890A Gas Chromatography/Agilent 5975C Mass Spectroscopic (GCMS) system (Agilent Technologies Inc., Beijing, China) equipped with an Agilent HP-5-MS capillary column $(30 \mathrm{~m} \times 0.25 \mathrm{~mm} \times 0.25 \mu \mathrm{m})$. The oven temperature was programmed using the method of Liang et al. [28]. Fatty acids were identified by comparison of the retention times to those of a standard fatty acid methyl esters mixture, and the results were expressed as percentage of total fatty acids. These measurements were carried out in triplicate.

\section{Lipidomics profiling}

The lipid extraction was performed according to Folch and Bligh methods $[29,30]$ with minor modification. Ground buckwheat $(60 \mathrm{mg})$ and chloroform-methanol-water $(\mathrm{v} / \mathrm{v} / \mathrm{v}=2: 1: 1,600 \mu \mathrm{L})$ were sonicated for $10 \mathrm{~min}$ and kept in freezer $\left(-20^{\circ} \mathrm{C}\right)$ for $20 \mathrm{~min}$, followed by frozen centrifugation at $14,000 \mathrm{~g}$ for $10 \mathrm{~min}$. The lipid-containing chloroform layer was collected and the residue was extracted twice more. The chloroform layer was combined and evaporated with $\mathrm{N}_{2}$. The dried lipid residue was redissolved in $200 \mu \mathrm{L}$ of methanol-isopropanol $(\mathrm{v} / \mathrm{v}=1: 1)$ and filtered through $0.22 \mu \mathrm{m}$ organic filter before analysis.

The lipidomics profiling was accomplished using a Nexera UPLC system (Shimadzu, Kyoto, Japan) tandem Q-Exactive Orbitrap mass spectrometer (Thermo Fisher, CA, USA) equipped with a heated electrospray ionization (HESI) probe, and separated on an ACQUITY UPLC BEH C18 column $(1.7 \mu \mathrm{m}, 2.1 \times$ 
$100 \mathrm{~mm}$ ) (Waters) in both negative and positive ionization modes. The binary solvent system consisted of mobile phase (A) acetonitrile-water $=6: 4$ and mobile phase $(B)$ acetonitrile-isopropanol $=1: 9$ (both mobile phase containing $0.1 \%$ formic acid and $10 \mathrm{mM}$ ammonium formate, $\mathrm{v} / \mathrm{v}$ ), and separation was achieved using the following gradient at a flow rate of $0.35 \mathrm{~mL} / \mathrm{min}: 30 \% \mathrm{~B}$ hold $3 \mathrm{~min}, 30-62 \% \mathrm{~B}$ over 3-5 min, 62-82\% B over 5-15 min, 82-99\% B over 15-16.5 min, the composition was held at $99 \%$ B for $1.5 \mathrm{~min}$, then $18-18.1 \mathrm{~min}, 99 \%$ to $30 \% \mathrm{~B}$, and $18.1-22 \mathrm{~min}$ holding at $30 \% \mathrm{~B}$. The injection volume was 5 $\mu \mathrm{L}$ and the mass range was set at $\mathrm{m} / \mathrm{z} 120-1800$. The HESI source parameters were set as follows: heater temperature of $300^{\circ} \mathrm{C}$; sheath gas flow rate of $45 \mathrm{Arb}$; auxiliary gas flow rate of $15 \mathrm{Arb}$; sweep gas flow rate of $1 \mathrm{Arb}$; spray voltage of $3.5 \mathrm{KV}$; capillary temperature of $320^{\circ} \mathrm{C}$. Lipid identification were performed using the software Lipidsearch 4.0 (Thermo Fisher, CA). According to the information for retention time and characteristic product ions, all lipids were identified based on MS1 (mass error < 5 ppm) and MS2 (mass error $<8$ ppm).

\section{Statistical analysis}

Data were statistically analyzed by one-way analysis of variance (ANOVA) using SPSS version 18.0 software (SSPS Institute, Chicago, USA). Duncan's multiple range test $(P<0.05)$ was performed to determine the significant differences. The correlation among the studied variables (lipase activity, FFA and content of each lipid subclass) in the samples was determined by two-tailed Pearson correlation analysis $(P<0.01)$. The linear regression of FFA vs. lipase activity was performed using Origin software (version 8.5, Microcal Inc., Northampton, MA, USA).

\section{Results}

\section{Effect of superheated steam on moisture content}

Figure 1a shows the effect of SS processing temperature and time on moisture content of buckwheat grains. The moisture content of SS-treated buckwheat grains decreased with the extended time and increased temperature of SS. When buckwheat grains were treated by $110^{\circ} \mathrm{C} \mathrm{SS}, 140^{\circ} \mathrm{C} \mathrm{SS}, 170^{\circ} \mathrm{C} \mathrm{SS}$ and $200{ }^{\circ} \mathrm{C}$ SS for $7 \mathrm{~min}$, their moisture contents were decreased from $20.26-14.82 \%, 12.10 \%, 8.89 \%$ and $5.81 \%$ respectively, namely, higher temperature led to higher rate of moisture removal of buckwheat grains. In particular, after treated with SS at $170^{\circ} \mathrm{C}$ for $5 \mathrm{~min}$, the moisture content decreased from $20.26-12.09 \%$, which was not significantly different from the original moisture content of untreated buckwheat (12.28\%).

\section{Effect of superheated steam on lipase inactivation}

Lipase activity of buckwheat grains decreased with an increase in processing time and temperature of SS (Fig. 1b). Higher temperatures $\left(170^{\circ} \mathrm{C}\right.$ and $\left.200^{\circ} \mathrm{C}\right)$ of SS treatment showed a better inactivate effect than $110^{\circ} \mathrm{C}$ or $140{ }^{\circ} \mathrm{C}$. An average level of lipase activity of buckwheat grains decreased by $26.24 \%, 28.98 \%$, $55.53 \%$ and $62.70 \%$, respectively, after the SS treatment at $110{ }^{\circ} \mathrm{C}, 140{ }^{\circ} \mathrm{C}, 170{ }^{\circ} \mathrm{C}$ and $200{ }^{\circ} \mathrm{C}$ for $7 \mathrm{~min}$. 
Meanwhile, it was observed that when SS treatment time was shorter than $1 \mathrm{~min}$, the steam temperature had no significant effect on the improvement of lipase inactivated efficiency. More than $50 \%$ of the lipase activity could be inactivated at $170^{\circ} \mathrm{C}$ when the SS treatment was more than $5 \mathrm{~min}$, but there was no significant difference between $170^{\circ} \mathrm{C}-5 \mathrm{~min}$ and $170^{\circ} \mathrm{C}-7 \mathrm{~min}$ in the reduction of lipase activity.

\section{Free fatty acid accumulation in buckwheat during storage}

The effect of SS on FFA accumulation during storage was shown in Fig. 2a-c. There was an increasing trend of FFA in untreated buckwheat at 4 and $25^{\circ} \mathrm{C}$ during storage, and the highest FFA was observed after 12 weeks of storage. The FFA of untreated buckwheat stored at $50{ }^{\circ} \mathrm{C}$ increased rapidly at the beginning of storage, reached maximum levels $(134.03 \mathrm{mg} \mathrm{KOH} / 100 \mathrm{~g} \mathrm{d.b}$.) at 8 weeks, and then showed a slight decline afterward. Compared with untreated buckwheat, SS-treated buckwheat exhibited significantly fewer accumulation in FFA during storage at each storage temperature. The FFA of SStreated buckwheat did not change significantly when stored at $4{ }^{\circ} \mathrm{C}$ and $25^{\circ} \mathrm{C}$ for 12 weeks, even for $50^{\circ} \mathrm{C}$ storage, which was always lower than $100 \mathrm{mg} \mathrm{KOH} / 100 \mathrm{~g}$ d.b.

\section{Changes in lipase of buckwheat during storage}

Changes in lipase of untreated and SS-treated buckwheat during storage were summarized in Fig. 2e-f. In general, the lipase activity of SS-treated samples was significantly $(P<0.05)$ lower than that of untreated samples at each temperature. The lipase of all untreated buckwheat samples initially decreased from $7.57 \mathrm{KOH} / \mathrm{g}$ d.b and reached minimum levels of $3.16-5.84 \mathrm{KOH} / \mathrm{g}$ d.b after 8 weeks of storage. The lipase activity of untreated buckwheat decreased most by $57.85 \%$ at storage temperature of $50{ }^{\circ} \mathrm{C}$ for 8 weeks, but that of SS-treated buckwheat decreased slightly lower by $44.44 \%$. Moreover, there was no significant difference in lipase activity of SS-treated buckwheat among different temperature conditions at the end of storage.

\section{Fatty acid compositions of buckwheat during storage}

Fatty acid compositions of untreated and SS-treated buckwheat during storage were summarized in Table 1. Before storage, the predominant fatty acids in untreated buckwheat were palmitic acid (18.04 \pm $0.35 \%)$, oleic acid $(34.31 \pm 0.43 \%$ ) and linoleic acid ( $35.00 \pm 0.58 \%$ ), which together constituted $87.35 \%$ of the total fatty acids. The percentages of stearic acid, behenic acid and docosenoic acid were significantly $(P<0.05)$ increased after SS treatment. There was no significant difference $(P>0.05)$ in percentage distribution of total SFA, total monounsaturated fatty acids (MUFA), and total polyunsaturated fatty acids (PUFA) between untreated and SS-treated buckwheat. After 12 weeks storage, the percentage of total SFA in untreated buckwheat increased significantly and that of total PUFA decreased significantly. The biggest reduction of PUFA was found in linoleic acid. However, SS treatment significantly retard the increase of SFA (mainly palmitic acid, stearic acid, arachidic acid and behenic acid) and the reduction of 
PUFA (mainly linoleic acid) during storage. The fatty acid compositions of stored SS-treated buckwheat were similar to those of fresh untreated and SS-treated buckwheat.

Table 1

Fatty acid compositions (\% of total fatty acids) of buckwheat grains during storage

\begin{tabular}{|c|c|c|c|c|}
\hline \multirow[t]{2}{*}{ Fatty acids } & \multicolumn{2}{|l|}{0 week } & \multicolumn{2}{|l|}{12 week } \\
\hline & Untreated & SS-treated & Untreated & SS-treated \\
\hline C14:0 Myristic & $0.16 \pm 0.14 a$ & $0.17 \pm 0.07 a$ & $0.16 \pm 0.06 a$ & $0.18 \pm 0.02 a$ \\
\hline C15:0 Pentadecanoic & $0.16 \pm 0.01 a$ & $0.13 \pm 0.02 b$ & $0.16 \pm 0.02 a$ & $0.14 \pm 0.00 a b$ \\
\hline C16:0 Palmitic & $18.04 \pm 0.35 b$ & $17.51 \pm 0.14 b$ & $19.04 \pm 0.65 a$ & $17.85 \pm 0.79 b$ \\
\hline C16:1 Palmitoleic & $0.21 \pm 0.06 b$ & $0.17 \pm 0.00 b$ & $0.17 \pm 0.00 b$ & $0.28 \pm 0.01 a$ \\
\hline C18:0 Stearic & $2.06 \pm 0.04 c$ & $2.14 \pm 0.01 b$ & $2.49 \pm 0.05 a$ & $2.00 \pm 0.02 c$ \\
\hline C18:1 Oleic & $34.31 \pm 0.43 a$ & $33.96 \pm 0.28 a$ & $34.02 \pm 0.35 a$ & $34.62 \pm 0.37 a$ \\
\hline C18:2 Linoleic ( $\omega-6)$ & $35.00 \pm 0.58 a$ & $34.91 \pm 0.30 a$ & $33.04 \pm 0.12 b$ & $34.83 \pm 0.29 a$ \\
\hline C18:3 Linolenic $(\omega-3)$ & $2.85 \pm 0.04 a$ & $2.72 \pm 0.04 a$ & $2.43 \pm 0.03 b$ & $2.47 \pm 0.17 b$ \\
\hline C20:0 Arachidic & $1.32 \pm 0.15 b$ & $1.50 \pm 0.13 b$ & $1.86 \pm 0.05 a$ & $1.41 \pm 0.06 b$ \\
\hline C20:1 Gadoleic & $2.84 \pm 0.12 a b$ & $3.07 \pm 0.06 a$ & $2.64 \pm 0.09 b$ & $2.73 \pm 0.20 b$ \\
\hline C22:0 Behenic & $1.69 \pm 0.11 c$ & $1.97 \pm 0.01 b$ & $2.41 \pm 0.09 a$ & $1.94 \pm 0.11 b$ \\
\hline C22:1 Docosenoic & $0.07 \pm 0.12 b$ & $0.25 \pm 0.01 a$ & $0.14 \pm 0.12 a b$ & $0.23 \pm 0.01 a b$ \\
\hline C24:0 Lignoceric & $1.29 \pm 0.19 a$ & $1.49 \pm 0.08 a$ & $1.44 \pm 0.05 a$ & $1.32 \pm 0.10 \mathrm{a}$ \\
\hline$\sum$ SFA & $24.72 \pm 0.15 b$ & $24.92 \pm 0.18 b$ & $27.56 \pm 0.49 a$ & $24.84 \pm 0.59 b$ \\
\hline$\sum$ MUFA & $37.43 \pm 0.45 a$ & $37.45 \pm 0.35 a$ & $36.97 \pm 0.56 a$ & $37.86 \pm 0.55 a$ \\
\hline$\sum$ PUFA & $37.85 \pm 0.58 a$ & $37.63 \pm 0.34 a$ & $35.47 \pm 0.09 b$ & $37.31 \pm 0.29 a$ \\
\hline $\begin{array}{l}\text { Values are mean } \pm \text { sta } \\
\text { statistical difference } \\
\text { polyunsaturated fatty }\end{array}$ & $\begin{array}{l}\text { d deviation ( } r \\
\text { 05). SFA, sat } \\
\text { s. }\end{array}$ & Mean in a ro & ferent lowerc & $\begin{array}{l}\text { tters indicate } \\
\text { ty acids; PUFA }\end{array}$ \\
\hline
\end{tabular}

\section{Lipidomics profile differences of buckwheat samples}

Figure S1 (Supplementary Material 1) shows representative UPLC-Q-Exactive Orbitrap MS spectra of lipid extract from different buckwheat samples. The signals in positive ionization mode were mainly attributed to sphingoid (So), phosphatidylcholine (PC), phosphatidylethanolamine (PE), triacylglycerol (TG), lysophosphatidylcholine (LPC), diacylglycerol (DG), and in negative ionization mode were mainly 
attributed to ceramide (Cer), cardiolipin (CL), phosphatidic acid (PA), phosphatidylglycerol (PG), phosphatidylserine (PS), phosphatidylinositol (PI), monogalactosyl diacylcerol (MGDG), digalactosyl diacylglycerol (DGDG). The different molecular species of lipids in buckwheat were shown in Tables S1S4 (Supplementary Material 2). A total of 457 lipid molecular species belonging to 23 lipid classes were detected. The lipids in buckwheat were dominantly composed of GPs and GLs, which accounted for $67.57-87.59 \%$ of the total lipids. The number of lipid molecular species of each lipid subclass in buckwheat was shown in Table 2. The sum of lipid molecular species of the buckwheat were: stored SStreated buckwheat $>$ fresh SS-treated buckwheat $>$ stored untreated buckwheat $>$ fresh untreated buckwheat. Before storage, the total species of GPs decreased and that of GLs increased after SS processing. Storage significantly increased the GLs and GPs species in untreated buckwheat, and SS treatment slow down this change in GPs species during storage. The lipids categories were analyzed by summing the relative abundance of individual lipids in the same class (Fig. 3). The relative abundance of $P C$ and PE decreased significantly after SS treatment before storage, and that of PS, PG, So, phosphatidylinositol monophosphate (PIP), lysophosphatidic acid (LPA), TG, DG and monoglyceride (MG) increased significantly $(P<0.05)$. Meanwhile, the relative abundance of PA, PC, LPA, digalactosyl monoacylglycerol (DGMG) and TG significantly decreased after storage, and that of CL, DGDG, sulfoquinovosyl diacylglycerol (SQDG), phosphatidylinositol diphosphate (PIP2), PE, PG and PS increased. However, SS treatment could retard the changes of SQDG, PE, PS and LPA during storage. It was noteworthy that the relative abundance of TG, DG and MG in stored SS-treated buckwheat decreased significantly, while that of monogalactosyl monoacylglycerol (MGMG), MGDG, DGMG and DGDG significantly increased, compared with the fresh untreated buckwheat. 
Table 2

The number of lipid molecular species of each lipid subclass in buckwheat grains during storage

\begin{tabular}{|c|c|c|c|c|c|}
\hline \multirow[t]{2}{*}{ Lipid classes } & \multirow[t]{2}{*}{ RT(time) } & \multicolumn{2}{|l|}{0 week } & \multicolumn{2}{|l|}{12 week } \\
\hline & & Untreated & SS-treated & Untreated & SS-treated \\
\hline LPA & $2.16-3.08$ & 3 & 3 & ND & 3 \\
\hline LPC & $2.01-4.37$ & 3 & 3 & 2 & 8 \\
\hline LPE & $4.42-6.83$ & 3 & 3 & ND & 1 \\
\hline PA & $7.50-12.47$ & 5 & 5 & 6 & 6 \\
\hline PC & $6.74-12.58$ & 26 & 8 & 25 & 19 \\
\hline PE & $2.54-12.85$ & 25 & 5 & 23 & 13 \\
\hline$P G$ & $4.57-13.16$ & 10 & 14 & 20 & 11 \\
\hline PS & $6.19-12.66$ & 11 & 16 & 17 & 13 \\
\hline $\mathrm{PI}$ & $6.43-12.99$ & 7 & 11 & 9 & 12 \\
\hline $\mathrm{CL}$ & $7.22-13.71$ & 10 & 12 & 18 & 17 \\
\hline PIP & $6.62-12.08$ & 6 & 9 & 5 & 9 \\
\hline PIP2 & $9.65-9.85$ & ND & ND & 2 & ND \\
\hline PIP3 & 8.67 & 1 & 1 & 1 & 1 \\
\hline Total GPs & $2.01-13.71$ & 109 & 89 & 127 & 112 \\
\hline DGDG & $6.27-11.75$ & 5 & 6 & 7 & 10 \\
\hline DGMG & $1.62-3.19$ & 3 & 3 & 1 & 3 \\
\hline MGDG & $4.09-11.65$ & 6 & 7 & 6 & 13 \\
\hline MGMG & $2.23-3.80$ & 1 & 1 & 2 & 3 \\
\hline SQDG & $2.35-11.63$ & 7 & 9 & 14 & 7 \\
\hline MG & $11.75-12.09$ & ND & 2 & ND & 2 \\
\hline DG & $7.21-15.71$ & 19 & 25 & 19 & 24 \\
\hline TG & $6.57-16.08$ & 90 & 135 & 95 & 123 \\
\hline Total GLs & $1.62-16.08$ & 131 & 188 & 144 & 185 \\
\hline
\end{tabular}

ND means not detected. 


\begin{tabular}{|lllllll|}
\hline Lipid classes & RT(time) & \multicolumn{2}{l}{ O week } & & \multicolumn{2}{l|}{ 12 week } \\
\cline { 3 - 4 } \cline { 6 - 7 } & & Untreated & SS-treated & & Untreated & SS-treated \\
\hline Cer & $1.79-13.35$ & 19 & 16 & & 15 & 17 \\
\hline So & $0.71-4.29$ & 5 & 6 & & 7 & 8 \\
\hline Total SPs & $0.71-13.35$ & 24 & 22 & & 22 & 25 \\
\hline SUM & & 264 & 299 & & 293 & 322 \\
\hline ND means not detected. & & & & \\
\hline
\end{tabular}

Association of hydrolytic rancidity with lipase activity and lipidomics profile The scatter plots and linear regression of FFA value vs. lipase activity in untreated and SS-treated buckwheat are presented in Fig. $4 a$ and b. The association of FFA value with lipase activity in untreated and SS-treated buckwheat had a $\mathrm{R}^{2}$ values of 0.8221 and 0.6777 , respectively. The slopes of SS-treated buckwheat (-8.28) was lower than that of untreated buckwheat (-10.27). The correlations of hydrolytic rancidity and lipid profiles were analyzed using Pearson correlation analysis and the correlation coefficients were given in Fig. 4c. The results showed that FFA value was significantly positively $(P<0.01)$ correlated with the relative abundance of SQDG, PE and PIP2, while had negative correlation with LPE ( $P$ $<0.01), T G, D G$ and MG. In addition, lipase activity was negatively correlated with the relative abundance of So, CL, MGMG, MGDG DGDG, SQDG, PA, PG, PI, PIP and PIP3.

\section{Discussion}

The aim of this study were to investigate the real effects of SS treatment on lipase inactivation, hydrolytic rancidity and lipidomics profile of buckwheat, and to verify the hypothesis that SS treated buckwheat grains prior to storage would inactivate lipase and thus stabilize buckwheat during storage. The study firstly optimize the best stabilization condition by SS treatment as $170^{\circ} \mathrm{C}-5 \mathrm{~min}$, based on the retaining moisture and relative high lipase inactivation in buckwheat grains, for the following storage experiments. Tempering prior to SS treatment was observed as an efficient way to eliminate enzymes in buckwheat grains, because it increased the moisture content and protected buckwheat from drying to very low final moisture content after SS processing. SS processing at $170^{\circ} \mathrm{C}-5 \mathrm{~min}$ was better for retaining moisture of buckwheat grains at $12.09 \%$. A moisture content of $16 \%$ is considered stable for avoiding buckwheat deterioration during storage [31], accordingly, buckwheat grains treated with $170{ }^{\circ} \mathrm{C}-5 \mathrm{~min}$ SS could be stored directly without drying or other additional moisture adjustment. Meanwhile, higher temperature and longer treatment of SS could improve the efficiency of lipase inactivation, and more than $50 \%$ lipase activity could be inactivated at $170{ }^{\circ} \mathrm{C}$ for $5 \mathrm{~min}$. Although the efficiency of $200^{\circ} \mathrm{C} \mathrm{SS}$ was more significant than $170{ }^{\circ} \mathrm{C}$, when the treatment time was extended to $5 \mathrm{~min}$ or more, the color of buckwheat grains changed greatly due to a mass loss of water within short processing time and thus partial scorch, which seriously affected the quality and acceptability. Moreover, lipase could not be completely 
inactivated in the present conditions, which might because the lipase in buckwheat was relatively stable compared with other grains, and the lipase activity in buckwheat was too low to reach a lower level. The lipase activity of oat (30 mg/g) was inactivated $78 \%$ by $160^{\circ} \mathrm{C}-2$ min SS treatment and inactivated completely at $170{ }^{\circ} \mathrm{C}-5 \mathrm{~min}$ [32]. The lipase activity of brown rice (5.25-12 mg/g) became constant at about $5 \%$ when the SS treatment time was extended to $2 \mathrm{~min}$, regardless of its temperature [24]. The present lipase results of buckwheat were in accordance to the previous study in highland barley that the lipase content in barley was too low to inactivated totally, and $160^{\circ} \mathrm{C}-8 \mathrm{~min}$ SS treatment could inactivated only $50 \%$ of lipase [33]. Whether SS could stabilize the quality of buckwheat by inactivating more than $50 \%$ lipase was determined in subsequent storage experiments.

Lipase hydrolysis results in the release of FFA and glycerol. Thus, an increase of the amount of FFA released from buckwheat grains during storage provides necessary information on potential development of hydrolytic rancidity. In this study, the lipidic acidity rates of untreated buckwheat significantly increased with an increase in storage period. The time to reach maximum level of FFA in the lipid hydrolysis process was related to enzyme activity and storage condition. Lipid oxidation and the inhibition of mould growth at extremely high storage temperature were key for the FFA inflection point of buckwheat at $50^{\circ} \mathrm{C}$ storage $[34,35]$. Similar results were reported by Li et al. [36] study that FFA value of brown rice initially increased at the beginning of storage and reached maximum levels after $225 \mathrm{~d}$ of storage, then followed by a slight decline. According to the GB/T35028-2018 [37], the FFA value of buckwheat flour with fresh and good quality must be below $120 \mathrm{mg} \mathrm{KOH} / 100 \mathrm{~g}$ d.b.. The FFA value in untreated buckwheat exceeded this prescribed limit seriously after stored at $50{ }^{\circ} \mathrm{C}$ for 8 weeks, accompanied with rancidity flavor. However, the SS-treated buckwheat could maintain good quality after 12 weeks of storage. SS treatment could retard FFA accumulation and inhibit lipid hydrolytic rancidity in buckwheat during storage, even SS processing was more effective than low temperature $\left(4^{\circ} \mathrm{C}\right)$ storage.

The inhibitory effect of SS treatment on lipid hydrolytic rancidity was also observed based on the lipase activity change during storage. The significantly lower lipase activity in SS-treated samples suggested that SS treatment could inactivate lipase and slow down the conversion rate of lipid, thus reduce the hydrolytic rancidity of lipid during storage. Meanwhile, the lower level of lipase variation in SS-treated buckwheat during storage, further indicated that inactivation of more than $50 \%$ lipase by SS could effectively stabilize the quality of buckwheat. SS could change the structures or conformations of lipase [38], so as to change the active site of the enzyme and the interaction with the substrate, and finally passivate the lipase activity and stabilize the quality of buckwheat.

The fatty acid composition was in accordance with FFA and lipase activity, supporting that SS treatment could suppress lipid hydrolysis and oxidation during storage. UFA accounted for a large proportion of fatty acids (75.28\%) in buckwheat, which was in accordance with the studies of lightly milled rice [15] and wheat bran [18]. Compared with SFA, UFA are important for functional properties of buckwheat and considered more beneficial to human health. However, UFA are highly susceptible to thermo-oxidation during heating due to the presence of $\pi$ bonds with high reactivity [15]. SS treatment was an oxygen-free heating medium, which protected UFA from oxidation. This is the reason why SS treatment significantly 
retard the increase of SFA (palmitic acid, stearic acid, arachidic acid, behenic acid) and the reduction of PUFA (linoleic acid) induced by storage. Maximum reduction in linoleic acid induced by storage indicated it might be the preferential substrate for oxidative rancidity [15]. These findings supported that SS processing was effective in suppressing lipid hydrolytic and oxidative rancidity while maintaining/improving buckwheat nutritional attributes and membrane stability during storage. Even though, SS treatment still caused slight lipid hydrolysis and changed lipid composition to some extent, according to the changes in fatty acid composition in fresh SS-treated buckwheat. It might be because heating treatment could result in disintegration of membranous structures or inactivation of heat labile antioxidants in cereals [39]. This was subsequently confirmed by the lipidomics profile results.

SS processing led to the decrease of total GPs species and the increase of total GLs species in buckwheat before storage, which was consistent with the previous results about changes in lipid molecule species by other thermal processing [40]. GPs and galactolipids are major structural constituents of cellular membranes and they play a key role in maintaining cellular homeostasis [41]. During the storage, the significant hydrolysis and oxidation of GPs and GLs occurred, based on the results of increased GLs and GPs species in stored untreated buckwheat, which caused the changes in components and structure of the phospholipid membrane, and further led to the release of TG [2]. However, SS treatment could slow down the change of GPs (mainly PE, PS and LPA) during storage, which might be because of the phospholipase inactivation. The decrease in TG, DG and MG of stored SStreated buckwheat indicated that GLs partially hydrolyzed, probably due to the destruction of phospholipid membrane by SS treatment prior to storage. The destruction of phospholipid membrane resulted in the TG leak which could contact with lipases in the aleurone and germ tissues [2]. Although SS-treated samples contained sufficient lipase substrates (e.g. TG), the degree of lipid hydrolysis and rancidity was much lower than that of untreated samples. Therefore, the enzyme activity, action sites and environment were particularly important for the stabilization of SS-treated buckwheat during storage. It was necessary to balance the inactivation of lipase and the destruction of phospholipid membrane by heat treatment to achieve better storage effect. This was also supported by our above results of FFA value and lipase activity that SS treatment altered the lipase site where the interaction with the lipase substrate were changed so that reducing lipase activity to low enough to prevent excessive accumulation of fatty acids.

Maintaining membrane integrity of cereals by reducing the saturation of fatty acids and the synthesis of galactolipids (e.g. DGDG, MGDG, SQDG) plays a key role in their growth adaptation [41, 42]. The increase in MGMG, MGDG, DGMG and DGDG of SS-treated buckwheat, suggested that SS treatment affected lipid metabolism of buckwheat during storage by modifying lipid composition, thus inhibiting the destruction of phospholipid membrane, maintaining the integrity and fluidity of cell membrane, and finally improving the storage quality of buckwheat. In terms of the nutritional application, the increase content of galactolipids improved the nutritional value of lipids in buckwheat. Given no scientific studies have been conducted directly to confirm the lipidomics profile of buckwheat after SS processing and/or storage, the further research on lipid metabolism pathway of buckwheat is still needed to be illustrated in more details. 
Liner regression analysis of FFA value in response to lipase activity showed that SS suppressed the rate of hydrolytic rancidity as compared to untreated buckwheat. With each unit decrease of lipase activity, a lower unit of FFA response were found for these SS-treated buckwheat. Correlation analysis showed that the increase of FFA mainly comes from the hydrolysis of GL, while the increase of SQDG, PE and PIP2 could be used as a marker of lipid hydrolytic rancidity. These results suggested that the inactivation of lipase by SS could effectively reduce the lipid hydrolytic rancidity of buckwheat during storage, and the change in lipidomics profile was effective to characterize the change of buckwheat quality.

\section{Conclusion}

The present study focused on the effects of SS processing on lipase activity, lipid hydrolytic rancidity, and lipidomics profile of buckwheat. SS processing at $170{ }^{\circ} \mathrm{C}$ for $5 \mathrm{~min}$ was proved to be an effective method for buckwheat stabilization. Inactivation of more than $50 \%$ lipase by SS could effectively inhibit lipid hydrolytic rancidity of buckwheat during storage. SS processing considerably suppressed FFA accumulation, lipase activity variation, UFA oxidation, and GPs hydrolysis (mainly PE, PS and LPA) during storage. SS processing could improve membrane integrity and fluidity of buckwheat during storage by regulating the UFA/SFA ratio and the content of galactolipids (mainly SQDG, MGMG, MGDG, DGMG and DGDG). Moreover, the correlation analysis indicated that the lipase inactivation had an important effect on lipid metabolism. Therefore, SS processing should be a new efficient technology that could inactivate lipase of buckwheat, change its GLs and GPs subclass metabolism during storage while maintain its lipid nutrition at the same time, so as to improve the storage quality of buckwheat. In addition, it is also demonstrated that lipidomics analysis is a workable approach to monitor the dynamic changes in lipid characteristics of buckwheat during storage, which provides scientific support for buckwheat quality control.

\section{Abbreviations}

SS:superheated steam; FFA:free fatty acid; UFA:unsaturated fatty acids; SFA:saturated fatty acids; PUFA:polyunsaturated fatty acids; MUFA:monounsaturated fatty acids; Cer:ceramide; CL:cardiolipin; DG:diacylglycerol; DGDG:digalactosyl diacylglycerol; DGMG:digalactosyl monoacylglycerol; GL:glycerolipid; GP:glycerophospholipid; LPA:lysophosphatidic acid; LPC:lysophosphatidylcholine; MG:monoglyceride; MGDG:monogalactosyl diacylcerol; MGMG:monogalactosyl monoacylglycerol; TG:triacylglycerol; So:sphingoid; SQDG:sulfoquinovosyl diacylglycerol; PA:phosphatidic acid; PC:phosphatidylcholine; PE:phosphatidylethanolamine; PG:phosphatidylglycerol; PI:phosphatidylinositol; PS:phosphatidylserine; PIP:phosphatidylinositol monophosphate; PIP2:phosphatidylinositol diphosphate; PIP3:phosphatidylinositol triphosphate.

\section{Declarations}

\section{Competing interests}


The authors declare no competing interests.

\section{Funding}

This work was supported by the earmarked fund for China Agriculture Research System (grant number CARS-07-E-04).

\section{Authors' contributions}

Lijuan Wang, collected, elaborated the literature and drafted the manuscript. Libo Wang, provided the technical assistance. Qiu Ju, helped to draft the manuscript. Zaigui Li, conceptualized the idea. Both authors read and approved the final manuscript.

\section{Acknowledgements}

Not applicable.

\section{Availability of data and material}

The dataset supporting the conclusions of this article is included within the article.

\section{Ethics approval and consent to participate}

Not Applicable.

\section{Consent for publication}

Not Applicable.

\section{Author details}

${ }^{1}$ College of Food Science and Nutritional Engineering, China Agricultural University, 17 Qinghua Dong Lu, Haidian District, Beijing, 100083, China

${ }^{2}$ Institute of Food and Nutrition Development, Ministry of Agriculture, 12 Zhongguancun South Stree, Haidian District, Beijing, 100081, China 


\section{Supplementary information}

Supplementary Material 1: Figure S1.

Supplementary Material 2: Table S1-S4.

\section{References}

1. Fan Z. Buckwheat starch: Structures, properties, and applications. Trends Food Sci Tech. 2016;49:121-35.

2. Liu L, Waters DLE, Rose TJ, Bao J, King GJ. Phospholipids in rice: Significance in grain quality and health benefits: A review. Food Chem. 2013;139:1133-45.

3. Li Q, Liang X, Zhao L, Zhang Z, Xue X, Wang K, Wu L. UPLC-Q-Exactive Orbitrap/MS-Based Lipidomics Approach To Characterize Lipid Extracts from Bee Pollen and Their In Vitro AntiInflammatory Properties. J Agr Food Chem. 2017;65:6848-6840.

4. Fahy E, Shankar S, Alex H, Glass B, Merrill CK, Murphy AH, Raetz RC, Russell CRH, Yousuke DW, Walter S. S. A comprehensive classification system for lipids. Eur J Lipid Sci Tech. 2005;107:337-64.

5. Mukhamedova KS, Glushenkova Al. Natural Phosphonolipids. Chem Nat Compd+. 2000;36:329-41.

6. Daniela K. Lenka, Taylor, Michael, SchneiderUlrich, Massing. Health effects of dietary phospholipids. Lipids Health Dis. 2012;11:3.

7. Martemucci G, D'Alessandro AG. Fat content, energy value and fatty acid profile of donkey milk during lactation and implications for human nutrition. Lipids Health Dis. 2012;11:113.

8. Mazza G. Lipid content and fatty acid composition of buckwheat seed. Cereal Chem. 1988;62:2747.

9. Suzuki T, Honda Y, Mukasa Y, Kim S-J. Effects of lipase, lipoxygenase, peroxidase, and rutin on quality deteriorations in buckwheat flour. J Agr Food Chem. 2005;53:8400-5.

10. Mazza G. Buckwheat browning and color assessment. Cereal chem. 1986;63:361-4.

11. Suzuki T, Kim S-J, Mukasa Y, Morishita T, Noda T, Takigawa S, Hashimoto N, Yamauchi H, MatsuuraEndo $\mathrm{C}$. Effects of lipase, lipoxygenase, peroxidase and free fatty acids on volatile compound found in boiled buckwheat noodles. J Sci Food Agr. 2010;90:1232-7.

12. Casas-Godoy L, Gasteazoro F, Duquesne S, Bordes F, Marty A, Sandoval G. Lipases: An Overview. Methods Mol Biol. 2018;1835:181-238.

13. Doblado-Maldonado AF, Pike OA, Sweley JC, Rose DJ. Key issues and challenges in whole wheat flour milling and storage. J Cereal Sci. 2012;56:119-26.

14. Chen M-H, Bergman CJ, McClung AM. Hydrolytic rancidity and its association with phenolics in rice bran. Food Chem. 2019;285:485-91.

15. Wu J, Mcclements DJ, Chen J, Liu W, Luo S, Liu C. Improvement in storage stability of lightly milled rice using superheated steam processing. J Cereal Sci. 2016;71:130-7. 
16. Zhong Y, Tu Z, Liu C, Liu W, Xu X, Ai Y, Liu W, Chen J, Wu J. Effect of microwave irradiation on composition, structure and properties of rice (Oryza sativa L.) with different milling degrees. J Cereal Sci. 2013;58:228-33.

17. Irakli M, Kleisiaris F, Mygdalia A, Katsantonis D. Stabilization of rice bran and its effect on bioactive compounds content, antioxidant activity and storage stability during infrared radiation heating. J Cereal Sci. 2018;80:135-42.

18. Hu Y, Wang L, Li Z. Superheated steam treatment on wheat bran: Enzymes inactivation and nutritional attributes retention. LWT - Food Sci Technol. 2018;91:446-52.

19. Ding C, Khir R, Pan Z, Zhao L, Tu K, El-Mashad H, McHugh TH. Improvement in Shelf Life of Rough and Brown Rice Using Infrared Radiation Heating. Food Bioprocess Tech. 2015;8:1149-59.

20. Chungcharoen T, Prachayawarakorn S, Tungtrakul P, Soponronnarit S. Quality Attributes of Germinated High-Amylose and Waxy Rice in Superheated Steam and Hot Air Drying. Dry Technol. 2015;33:876-85.

21. Hu Y, Nie W, Hu X, Li Z. Microbial decontamination of wheat grain with superheated steam. Food Control. 2016;62:264-9.

22. Wu J, Chen J, Liu W, Liu C, Zhong Y, Luo D, Li Z, Huang Z. Selective peroxidase inactivation of lightly milled rice by superheated steam. J Cereal Sci. 2014;60:623-30.

23. Head D, Cenkowski S, Arntfield S, Henderson K. Storage stability of oat groats processed commercially and with superheated steam. LWT - Food Sci Technol. 2011;44:261-8.

24. Satou K, TAKAHASHI Y. YOSHII Y. Effect of Superheated Steam Treatment on Enzymes Related to Lipid Oxidation of Brown Rice. Food Sci Technol Res. 2010;16:93-7.

25. Head DS, Cenkowski S, Arntfield S, Henderson K. Superheated steam processing of oat groats. LWT Food Sci Technol. 2010;43:690-4.

26. Jha PK, Kudachikar VB, Kumar S. Lipase inactivation in wheat germ by gamma irradiation. Radiat Phys Chem. 2013;86:136-9.

27. Zhao S, Xiong S, Qiu C, Xu Y. Effect of microwaves on rice quality. J Stored Prod Res. 2007;43:496502.

28. Liang P, Li R, Sun H, Zhang M, Cheng W, Chen L, Cheng X, Akoh CC. Phospholipids composition and molecular species of large yellow croaker (Pseudosciaena crocea) roe. Food Chem. 2018;245:80611.

29. Folch J, Lees M, Stanley GHS. A simple method for the isolation and purification of total lipides from animal tissues. J Biol Chem. 1957;226:497-509.

30. Bligh EG, Dyer WJ. A rapid method of total lipid extraction and purification. Can J Biochem Physiol. 1959;37:911-7.

31. Pomeranz Y, Lorenz K. Buckwheat: Structure, composition, and utilization. C R C Crit Rev Food Technol. 1983; 19:213-258. 
32. Chang Y, Li X-P, Liu L, Ma Z, Hu X-Z, Zhao W-Q, Gao G-T. Effect of Processing in Superheated Steam on Surface Microbes and Enzyme Activity of Naked Oats. J Food Process Pres. 2015;39:2753-61.

33. Wang $\mathrm{H}$, Wang L, Tong L, Li Z. Effect of superheated steam inactivation on naturally existent microorganisms and enzymes of highland barley. Int J Food Sci Tech. 2019;54:2570-7.

34. Rani PR, Chelladurai V, Jayas DS, White NDG, Kavitha-Abirami CV. Storage studies on pinto beans under different moisture contents and temperature regimes. J Stored Prod Res. 2013;52:78-85.

35. Liu K, Li Y, Chen F, Yong F. Lipid oxidation of brown rice stored at different temperatures. Int J Food Sci Tech. 2017;52:188-95.

36. Li Y, Liu K, Chen F. Effect of selenium enrichment on the quality of germinated brown rice during storage. Food Chem. 2016;207:20-6.

37. SAMR-SA. State Administration for Market Regulation, and Standardization Administration of People's Republic of China. GB/T35028-2018 Buckwheat flour. 2018.

38. Qu C, Wang H, Liu S, Wang F, Liu C. Effects of microwave heating of wheat on its functional properties and accelerated storage. J Food Sci Technol. 2017;54:1-8.

39. Lehtinen P, Katja K, Ilkka L, Simo L. Effect of Heat Treatment on Lipid Stability in Processed Oats. J Cereal Sci. 2003;37:215-21.

40. Shi C, Guo H, Wu T, Tao N, Wang X, Zhong J. Effect of three types of thermal processing methods on the lipidomics profile of tilapia fillets by UPLC-Q-Extractive Orbitrap mass spectrometry. Food Chem. 2019;298:125029.

41. Peppino Margutti M, Reyna M, Vilchez AC, Villasuso AL. Lipid profiling shows tissue-specific differences in barley for glycerolipid composition in response to chilling. Environ Exp Bot. 2019;158:150-60.

42. Drmann P, Benning C. Galactolipids rule in seed plants. Trends Plant Sci. 2002;7:112-8.

\section{Figures}



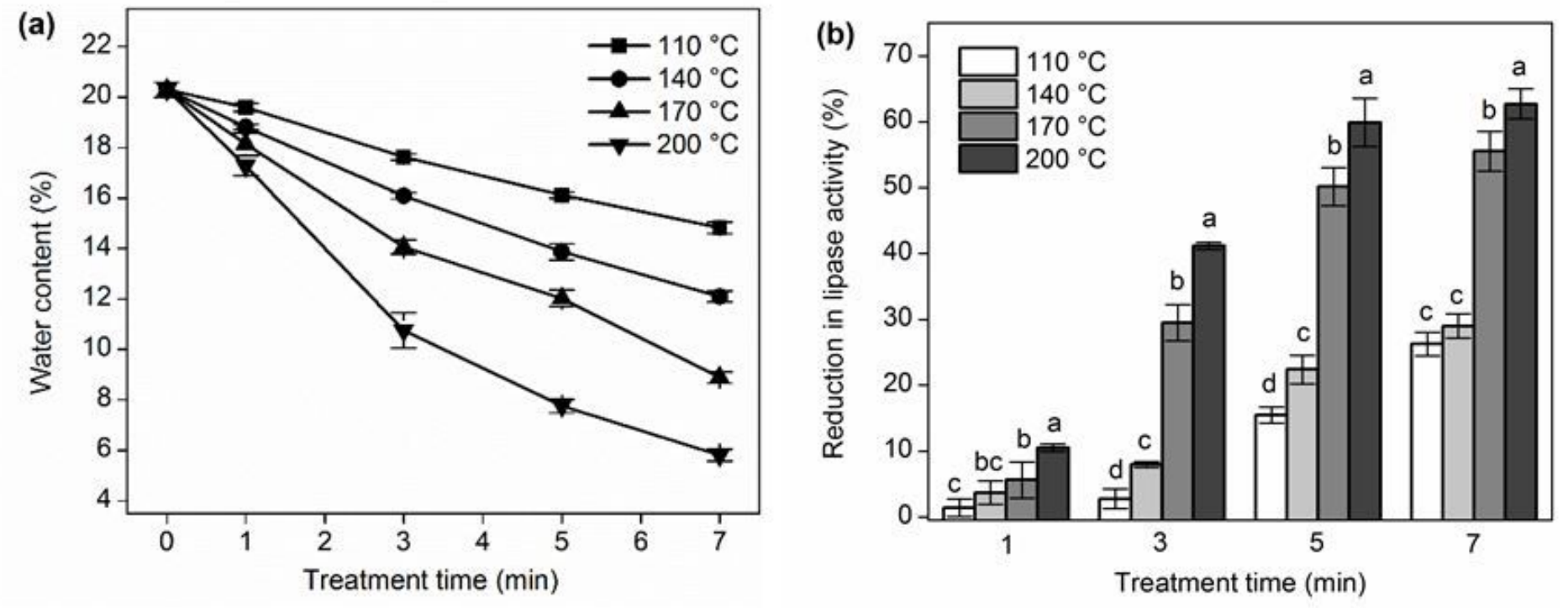

Figure 1

Effect of SS on moisture content (a) and lipase activity (b) of buckwheat grains. Values are expressed as mean \pm standard deviation $(n=3)$. Different lowercase letters marked above the bar indicate statistical difference $(P<0.05)$. 

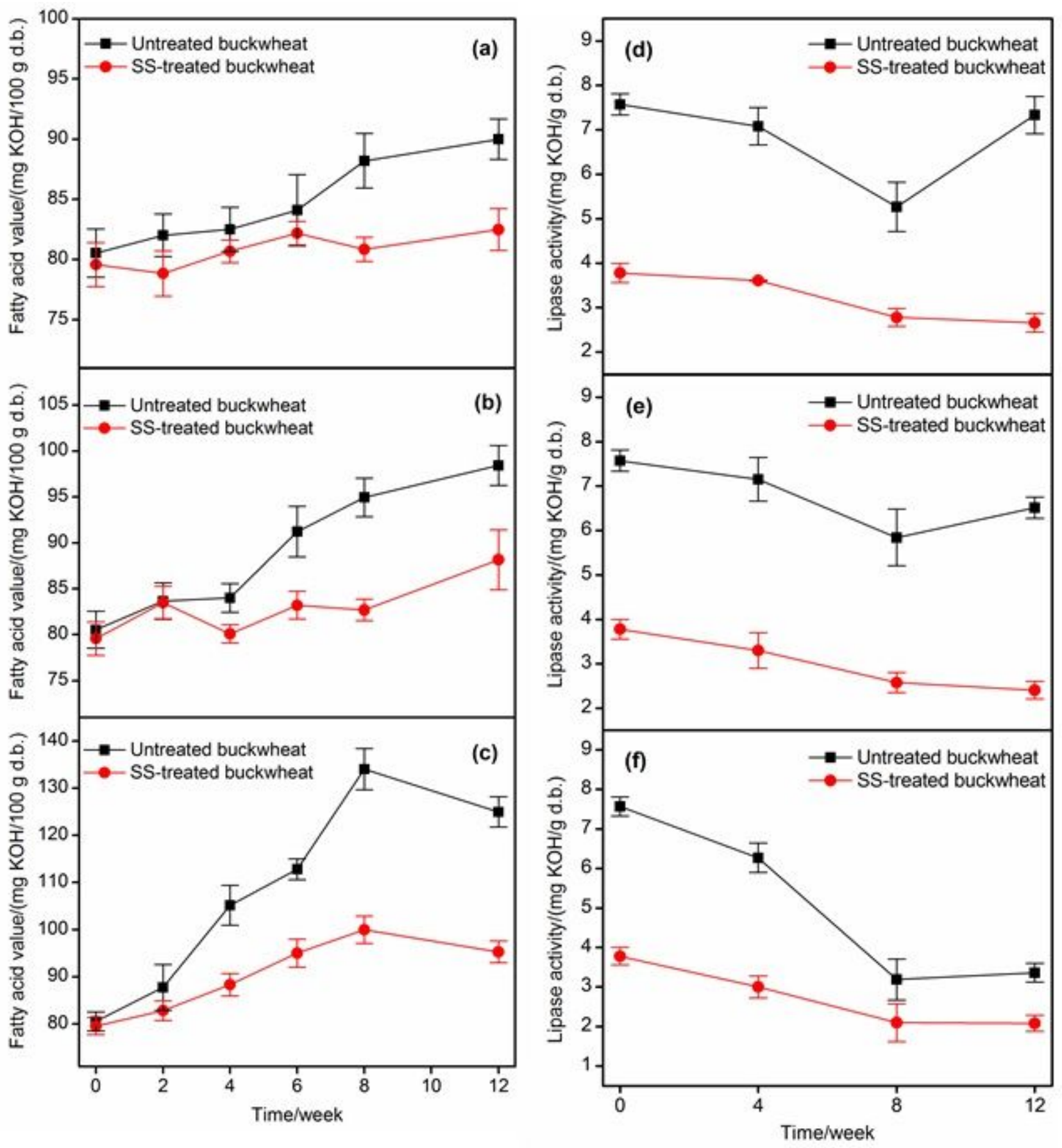

Figure 2

Changes of FFA value (a-c) and lipase activity (d-f) in buckwheat grains during storage. FFA at (a) 4, (b) 25 , and (c) $50{ }^{\circ} \mathrm{C}$. Lipase activity at (d) 4 , (e) 25 , and (f) $50^{\circ} \mathrm{C}$. Values are expressed as mean \pm standard deviation $(n=3)$. 

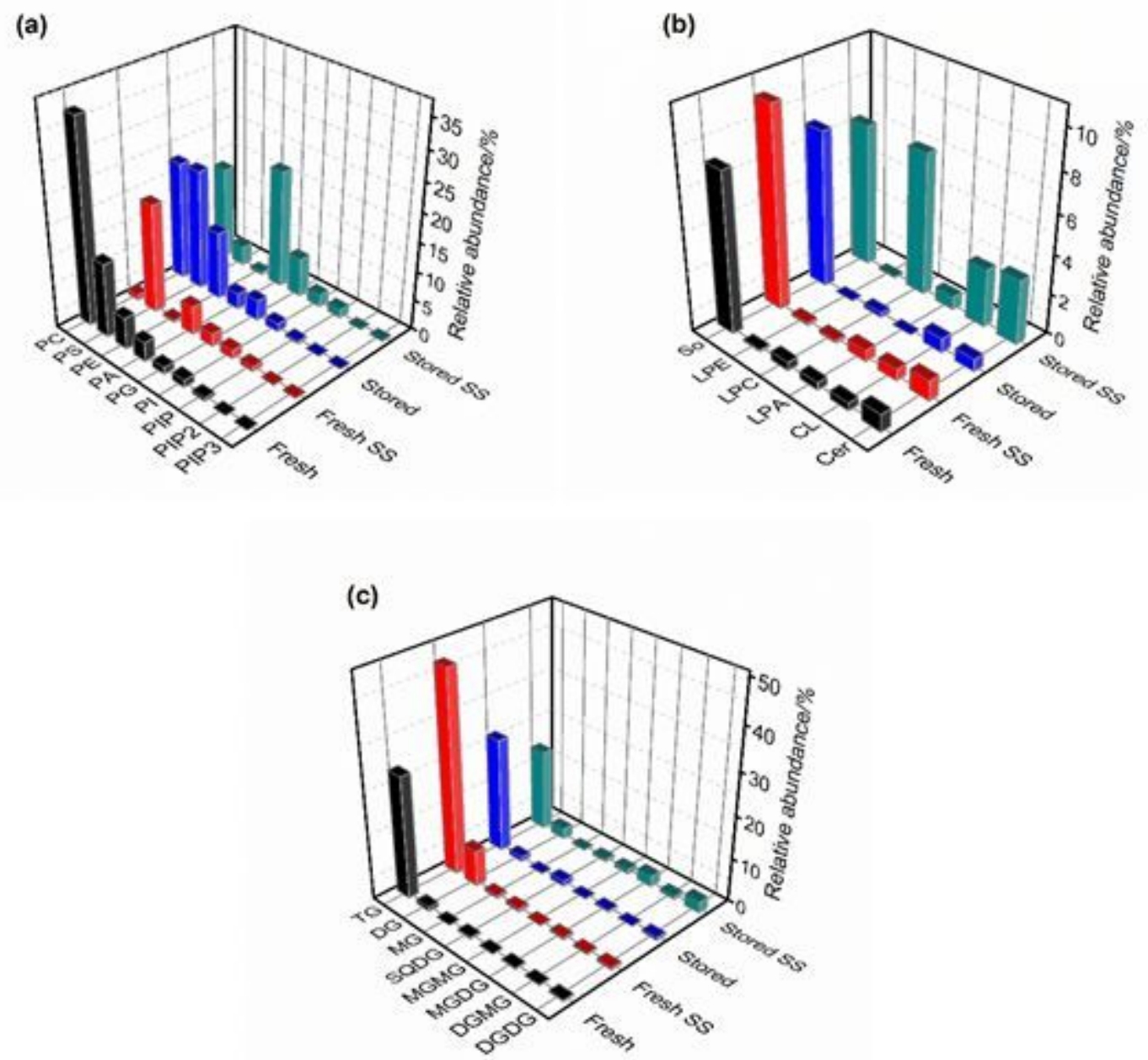

Figure 3

Relative abundance (\%) of each lipid subclass in buckwheat grains during storage. Fresh: untreated buckwheat at 0 week; Fresh SS: SS-treated buckwheat at 0 week; Stored: untreated buckwheat at 12 week; Stored SS: SS-treated buckwheat at 12 week. 


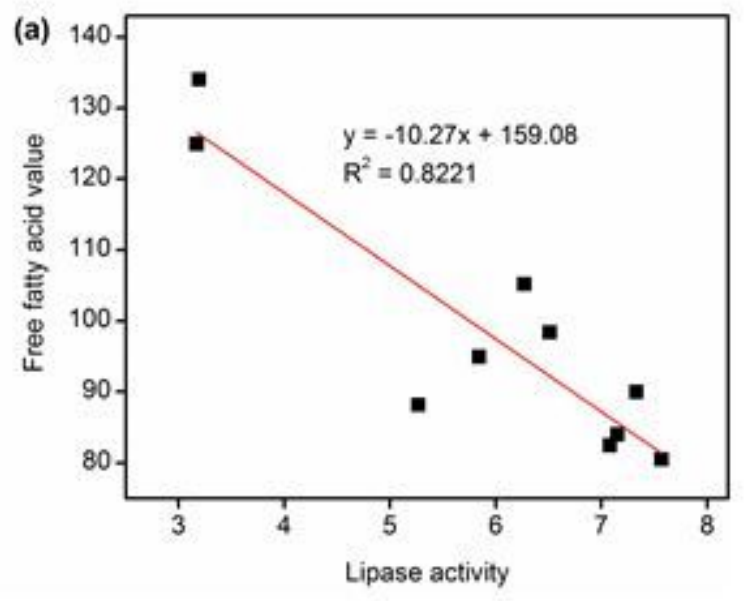

(c)

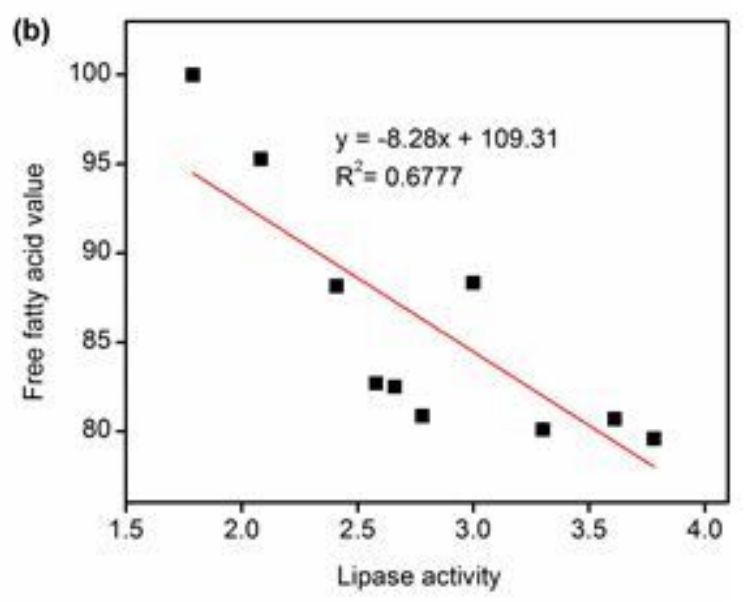

\begin{tabular}{|c|c|c|}
\hline & FFA & Lipase \\
\hline Cer & 0.07 & -0.56 \\
\hline So & 0.06 & $-0.78^{* *}$ \\
\hline $\mathrm{CL}$ & 0.2 & $-0.63^{*}$ \\
\hline DGDG & 0.19 & $-0.63^{\circ}$ \\
\hline DGMG & -0.04 & -0.53 \\
\hline MGDG & 0.12 & $-0.60^{*}$ \\
\hline MGMG & 0.11 & $-0.58^{*}$ \\
\hline SQDG & $0.97=0$ & $-0.72 * *$ \\
\hline LPA & -0.38 & -0.35 \\
\hline LPC & 0.12 & -0.56 \\
\hline LPE & $-0.95^{*}$ & 0.33 \\
\hline $\mathrm{PA}$ & 0.09 & $-0.58^{*}$ \\
\hline PC & 0.19 & 0.44 \\
\hline PE & $0.75^{e *}$ & 0.11 \\
\hline PG & 0.36 & $-0.73^{* *}$ \\
\hline PS & 0.36 & 0.07 \\
\hline PI & 0.21 & $-0.64^{*}$ \\
\hline PIP & 0.04 & $-0.60^{*}$ \\
\hline PIP2 & $0.880=$ & -0.26 \\
\hline PIP3 & 0.1 & $-0.59^{*}$ \\
\hline TG & -0.49 & -0.18 \\
\hline DG & -0.47 & -0.38 \\
\hline MG & -0.53 & -0.25 \\
\hline
\end{tabular}

Figure 4

Association of hydrolytic rancidity with lipase activity and lipidomics profile in buckwheat. Linear regression of FFA value and lipase activity in untreated buckwheat (a) and SS-treated buckwheat (b); Pearson correlation coefficients of hydrolytic rancidity and different lipid species (c). Data with single asterisk (*) and double asterisk (**) are statistically significant at $\mathrm{P}<0.05$ and $\mathrm{P}<0.01$, respectively.

\section{Supplementary Files}

This is a list of supplementary files associated with this preprint. Click to download.

- TableS1S4.docx

- Figures1.docx

- Personalcover.pdf

- Tables1S4.docx

- Figures1.docx

- Personalcover.pdf 
Page 23/23 